\title{
Apophis 2029: \\ Decadal Opportunity for the Science of Planetary Defense
}

\author{
White paper submitted to: \\ Planetary Science and Astrobiology Decadal Survey 2023-2032 \\ Richard P. Binzel \\ Massachusetts Institute of Technology \\ Cambridge, MA 02139 \\ rpb@mit.edu \\ 617-253-6486
}

Brent W. Barbee, NASA GSFC

Olivier S. Barnouin, JHU APL

James F. Bell, ASU

Mirel Birlan, Paris Obs.

Aaron Boley, U. British Columbia

William Bottke, SWRI

Marina Brozović, JPL

Joshua T. Cahill, JHU APL

Humberto Campins, Univ. Central Florida

Andy Cheng, JHU APL

Paul W. Chodas, JPL Terik Daly, JHU APL

William C. Danchi, NASA GSFC

Joseph V. DeMartini, Univ. Maryland

Francesca DeMeo, MIT

Daniel Durda, SWRI

Anthony Freeman, JPL

Masatoshi Hirabayashi, Auburn Univ.

Yaeji Kim, Auburn Univ.
Lucy F. Lim, NASA GSFC

Michael Marsset, MIT

Patrick Michel, Obs. Cote d'azure

Nicholas Moskovitz, Lowell Observatory

Michael C. Nolan, Univ. Arizona

Joseph A. Nuth III, NASA GSFC

Ryan S. Park, JPL

Alex H. Parker, SWRI

Carol A. Raymond, JPL

Kim R. Reh, JPL

Derek Richardson, Univ. Maryland

Paul Sava, Colorado School of Mines

Daniel J. Scheeres, Univ. Colorado

Damya Souami, Paris Obs.

Jean Souchay, CNRS, Sorbonne-Universités

Jessica M. Sunshine, Univ. Maryland

Patrick A. Taylor, Lunar Planetary Institute

Flaviane Venditti, Univ. Central Florida

Anne Virkki, Univ. Central Florida

Donald K. Yeomans, JPL/Retired 
Binzel et al. - Apophis 2029

\section{Apophis 2029: \\ Decadal Opportunity for the Science of Planetary Defense}

\section{Executive Summary:}

Many advancements in planetary science are achieved by seizing upon the opportunity created by rare natural events (e.g. comet Shoemaker-Levy 9 Jupiter impact; 1994) and by planned physical interactions (e.g. Deep Impact into comet Tempel 1; 2005). A forthcoming opportunity to seize upon a natural physical "experiment" occurs on April 13, 2029 with the unprecedentedly close Earth encounter by the large 340m asteroid (99942) Apophis. On that date, nature is performing the "experiment" of subjecting the physical body of Apophis to Earth's tidal torques as it approaches to within 31,000 km of Earth's surface, a distance that is closer than orbiting geosynchronous satellites.

A potentially hazardous asteroid (PHA) as large as Apophis encountering Earth this closely (within 0.1 lunar distances) is, on average, a once-per-thousand year event. (In context, Apophis is 7 times larger and 350 times more massive than the Tunguska 1908 impactor; 5000 times more massive than Chelyabinsk 2013.) Because of this event's incredible rarity, knowledge gained through measurements and outcomes of the Apophis 2029 "natural experiment" are clearly a decadal, if not millennial, opportunity for planetary science. Most specifically, this knowledge opportunity is for the science supporting planetary defense. Further, on April 13, 2029 all of Earth will be watching: Apophis will be visible to the naked eye speeding across the evening sky for an estimated 2 billion people spanning western Europe and northern Africa.

In this White Paper we outline our current best understanding, and uncertainties, for scientific advances in the physical study of potentially hazardous asteroids that may be achievable by measuring physical changes of Apophis' spin, surface structure, and/or shape configuration in response to Earth's tidal torques. If tidal torques themselves, or surface configuration changes induce any measurable seismic vibration signal inside Apophis, a new field of asteroid seismology has the potential to be born. Over six decades of planetary science, seismology has been achieved beyond Earth for only two planetary worlds: Moon and Mars.

With this White Paper, we take no position on how to implement specific investigations capable of achieving the science advances offered by the Apophis 2029 opportunity. Instead we advocate that competitive selection of investigations, be they theoretical or observational (ground-based, space-based, or in situ), be executed under the direction of NASA's Planetary Defense Coordination Office, with a projected and perhaps necessarily augmented budget capable of supporting them. International collaboration is strongly encouraged.

Thus, summarizing in four specific points, we urge the framers of the Decadal Survey to:

- Recognize the decadal, if not millennial, opportunity for the science of planetary defense presented by the Apophis 2029 once-per-thousand-year "natural experiment."

- Prioritize as a top-level planetary defense science goal modeling and measuring the physical outcome on Apophis exerted by Earth's tidal torques so as to achieve the greatest possible new insights into the physical nature, including the internal structure, of PHAs.

- Recognize that time is of the essence for defining and implementing investigations of physical effects on Apophis, particularly if in situ measurements are to be considered. An Apophis 2029 Science Definition Team may be prescribed.

- Recognize that the achievable knowledge of PHAs presented by the Apophis 2029 opportunity could have immeasurable benefits to the future of humanity, in the highly unlikely, but not impossible necessity to mitigate a future impact threat. 
Binzel et al. - Apophis 2029

\section{Apophis 2029: \\ Decadal Opportunity for the Science of Planetary Defense}

\section{Asteroid Close Encounters: Separating Fact from 'Media Sensation'}

Weekly, if not daily, news stories declare warnings of impending asteroid "close encounters" with Earth. While tabloids are easily dismissed, mainstream media outlets are not immune, even for objects passing more than 10 lunar distances away (Figure 1). The cacophony of asteroid sensationalism by the media dulls the context for truly spectacular close encounter opportunities.

Figure 2 displays a timespan of two centuries to give context for the frequency of known asteroid encounters within less than one-half lunar distance. Objects a few meters in size pass inside the lunar distance weekly, and the modern uptick in their discovery efficiency is apparent in the data. However, over this two-century window, one event spectacularly stands out.

\section{Apophis April 13, 2029: A Decadal If Not Millennial Close Encounter Opportunity}

As shown in Figure 2, the $340 \mathrm{~m}$ asteroid Apophis is the largest known object encountering Earth within 0.1 lunar distance over the span of two centuries (Brozović 2018). Apophis is 7 times larger and 350 times more massive than estimates for the Tunguska 1908 impacting body (Robertson \& Mathias 2019) and 5000 times more massive than Chelyabinsk (Popova 2013).

On April 13, 2029 (a Friday), Apophis approaches within 31,000 km of Earth's surface, a miss distance that is closer than orbiting geosynchronous satellites. Time averaged, an object as large as Apophis approaches Earth within 0.1 lunar distance approximately once-per-thousand years, making Apophis 2029 a once-per-millennium type of science opportunity. In fact, we can also expect that all of Earth will be watching. In the early evening hours of April 13, 2029 Apophis' rapid trek across the sky (brightening to $3^{\text {rd }}$ magnitude) will be visible to the unaided eye for an estimated 2 billion people spanning western Europe and northern Africa.

\section{Apophis 2029: Decadal Opportunity for the Science of Planetary Defense}

The science opportunity of the Apophis 2029 encounter arises not only because of the proximity for Earth observers. The Apophis 2029 encounter presents an unprecedented science opportunity because of the Earth's physical interactions on the body of Apophis itself. At its geocentric miss distance of six Earth radii, Apophis will pass well outside the canonical Roche limit ( $\sim 2$ planetary radii). Recall however, that the Roche limit describes destruction of a passing body. At a miss distance of six Earth radii, significant tidal torques will be exerted on the body of Apophis. The consequences of Earth's tidal torques on Apophis include altering its rotation state, with the possibility of creating internal structural alteration within the asteroid itself, possibly resulting in measurable seismic waves and real-time surface disturbances.

As discussed in Section 5, considerable uncertainty remains in predicting which, if any, of these observable effects will occur on Apophis and be measurable within the precision limits of applicable technology. The greatest uncertainty factor for the outcome is the lack of knowledge of the internal structure of potentially hazardous asteroids. Thus, we urge the framers of the Decadal Survey to recognize the Apophis 2029 encounter as a "natural experiment" whose measurable outcomes could be transformative in understanding the internal structure for PHAs.

Herein we argue that the Apophis 2029 encounter event has an historical planetary science parallel: The 1994 Jupiter impact by comet Shoemaker-Levy 9 (SL9). Significant resources were allocated and extensive observational campaigns were organized for reasons that included: i) the event was unprecedented to modern science and modern measurement technology; 


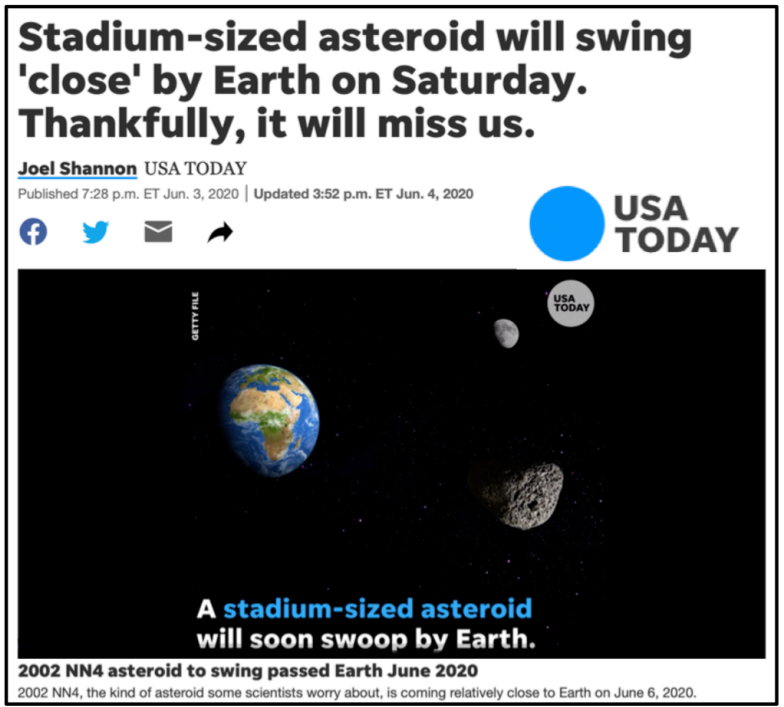

FIGURE 1: June 2020 example of mainstream media carrying "asteroid alert" stories that dull attention from meaningful close approach events. Despite the depiction shown, 2002 NN4 missed the Earth by >13 lunar distances.

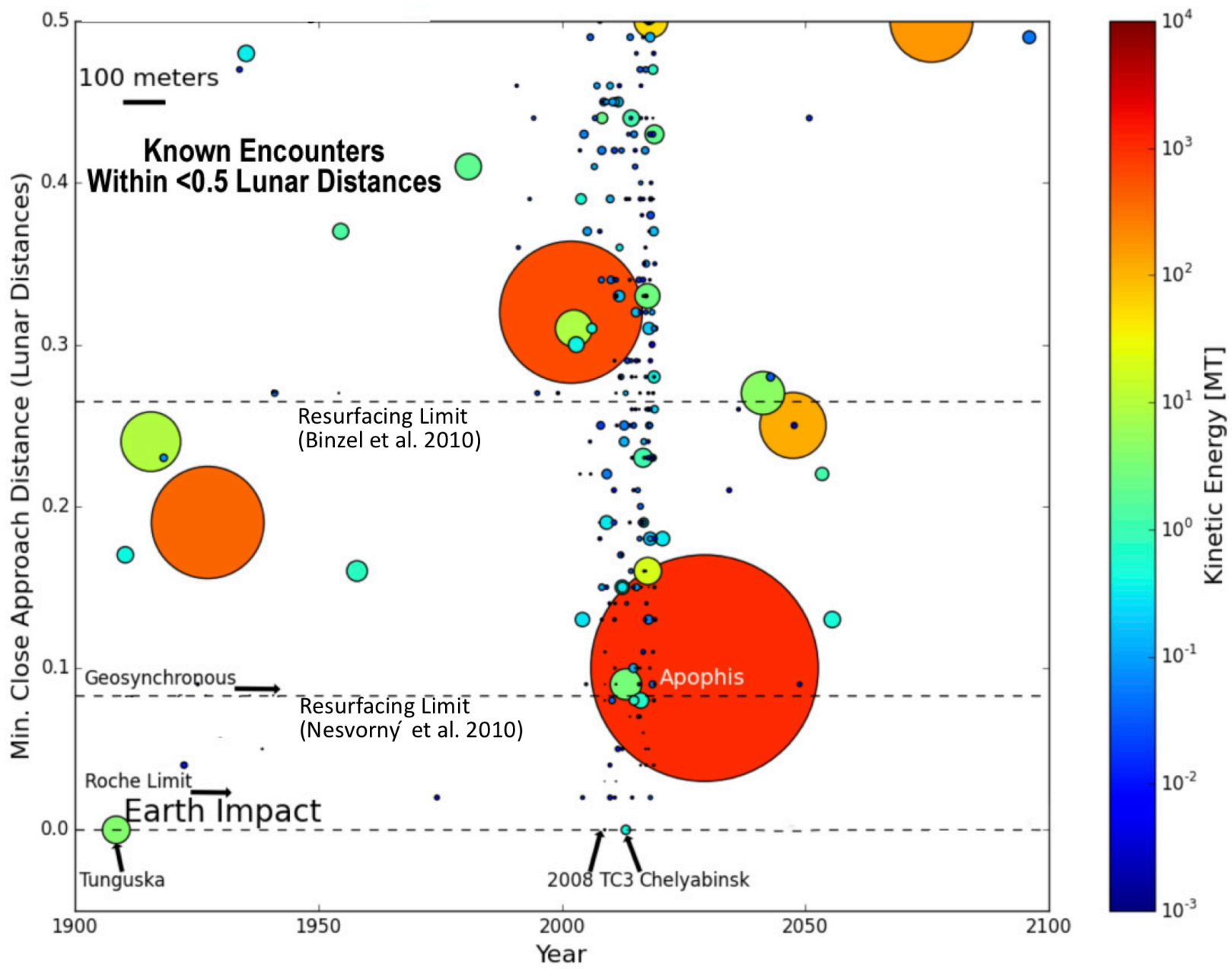

FIGURE 2: A two-century view (1900 to 2100) of known Earth-approaches within $1 / 2$ the lunar distance. Apophis 2029 stands out as an extremely rare event. Object sizes are depicted according to the scale bar, top left. For the "news story" object 2002 NN4 shown in Figure 1), its miss distance is off the scale of this figure by a factor of 25 .

[Figure from R. P. Binzel, A.M. Earle; Planetary Defense Conference 2019. Data are from NASA/JPL Center for NEO Studies https://cneos.jpl.nasa.gov/ca/, Low orbital certainty data omitted.] 
ii) the SL9 impact was a "natural experiment" whose outcome was unpredictable and ultimately surprising, see Table I; iii) detailed observations and measurements of the impact consequences promised (and delivered) interdisciplinary advances in understanding the structure of comets, the structure and chemistry of Jupiter's atmosphere, and impact physics. Further, the "impact" of SL9 on public and Congressional awareness of asteroid hazards cannot be understated.

Herein we also point out precedents for planetary science mission expenditures up to the level of Discovery Class missions for conducting active experiments: Deep Impact into Tempel 1; Double Asteroid Redirection Test (DART). For each, the mission motivation was driven by the potential new knowledge to be gained by resolving the wide-ranging uncertainty of the outcome. Thus, we argue for Apophis 2029: The uncertainty of the outcome creates a strong motivation to seize upon this unprecedented learning opportunity for insights into the physical characteristics of potentially hazardous asteroids. This argument also revolves around a central motivation for planetary defense itself: In the highly unlikely, but not impossible necessity to mitigate a future asteroid threat, the physical insights gained from the Apophis 2029 encounter could be one of the most important science products ever delivered to humanity by planetary science investigations.

\section{Implementation of Apophis 2029 Investigations}

Within this White Paper, we take no position on how to implement specific prediction or observational investigations capable of achieving measurement and science advances offered by the Apophis 2029 opportunity. (Rationale for in situ investigation are given by Barbee 2020; Cheng 2020. Taylor 2020 describes the essential role of radar; Haynes 2020 describes the potential of radar tomography.) As outlined in Section 5, predictions for measurable outcomes are highly uncertain at this time. We advocate the knowledge potential of the Apophis 2029 opportunity merits a directed Science Definition Team study. We note particularly that time is of the essence for considering in situ measurements. For example, a student-led study (MIT Project Apophis, 2017) identified low cost mission opportunities opening as early as 2026.

While planetary science expertise and techniques enable the path forward, we argue that the new knowledge gained has its greatest value to the science of planetary defense. In colloquial terms, Apophis is the "Poster Child for Planetary Defense." For this reason, we advocate that Apophis 2029 investigations be supported under NASA's Planetary Defense budget, in accordance with a specific recommendation of the Melosh (2019) National Academy Report:

"Missions meeting high-priority planetary defense objectives should not be required to compete against missions meeting high-priority science objectives."

We thus propose as a Decadal Report recommendation: Competitively selected or directed Apophis science investigations, be they theoretical or observational, ground-based, space-based, or in situ, be executed under the direction of NASA's Planetary Defense Coordination Office $(P D C O)$. Commensurate with this recommendation, an appropriate budget must be allocated within the NASA Science Mission Directorate that is capable of specifically supporting Apophis investigations. International collaboration is strongly encouraged. We note that the PDCO is currently supporting, and should be encouraged to continue support, international planning efforts toward the Apophis 2029 opportunity.

\section{Apophis 2029 Model Predictions, Science Implications, Measurements and Uncertainties}

While Apophis is coming for certain, and it will miss the Earth for certain, the actual physical consequences of the encounter on Apophis itself are uncertain. As noted in Section 3, the Apophis 2029 miss distance of 6 Earth radii is outside the canonical Roche limit (for destruction), but still close enough to subject the physical body of Apophis to tidal torques.

Table I summarizes a variety of physical effects that have been studied to date by a variety of independent models. \{Covid-19 Note: A more detailed compilation was to be a product of a 
planned Apophis T-9 Workshop in April 2020, postponed to November 2020.\} As a first table entry, the 2029 encounter will result in Apophis undergoing a definitive orbit change (semimajor axis altered from 0.9 AU to $>1$ AU; Farnocchia et al. 2013). Current models are also certain for a change occurring in Apophis' rotational properties (e.g. Scheeres et al. 2005; see Table I). Apophis is presently in a tumbling non-principal axis state with precession and rotation periods of 27.38h and 263h, respectively (Pravec et al. 2014; Brozović et al. 2018). The tumbling state of Apophis may be a result of torques exerted during one or more previous (but not calculable with precision) Earth encounters. As Apophis experiences new torques on its angular momentum vectors during the 2029 encounter, the physical (and observable) consequences of the new body axis misalignments remain uncertain (e.g. Yu et al. 2014). In the most substantial case, any re-arrangement of Apophis' (presumed) interior rubble pile structure could result in a gross reconfiguration of its shape. Models to date (e.g. Scheeres et al. 2020), however, deem that a gross reconfiguration is unlikely for such a "far" encounter outside of the canonical Roche limit.

Minor internal reconfigurations, or just the body's adjustment to a new rotation state, has the potential to spur downslope movement of regolith and/or generate seismic waves inside of Apophis (Souchay et al. 2014, 2018; Yu et al. 2014; DeMartini et al. 2019). Beyond Earth, natural seismic waves have been measured for only two planetary bodies in the history of solar system exploration: Moon by Apollo and Mars by InSight. In the context of priorities set by past Decadal Surveys for seismological investigations, and the considerable planetary mission expense allocated toward achieving these seismic measurements, we propose a specific finding:

The potential for measuring seismic waves in Apophis, thereby providing the first direct insight into the internal structure of a potentially hazardous asteroid, is the most enticing and transformative outcome that may be achievable through Apophis 2029 investigations.

Observational evidence supports finding that planetary encounters outside of the Roche limit generate enough (presumably) seismic activity to rearrange the upper surface layer (Binzel et al. 2010). Terentjeva (2014) suggests a possibly associated meteor stream. "Refreshing" by Earth encounters was suggested by Nesvorny' et al. (2005) and subsequently modeled by Nesvorny' et al. (2010). The 'seismic limit' inside which the encounter is close enough to effect surface changes is uncertain, with Binzel et al. (2010) finding spectrally fresh surfaces consistent with a limiting distance inside of $\sim 15$ Earth radii. More detailed theoretical modeling by Nesvorny' et al. (2010) places the 'seismic limit' inside of $\sim 5$ Earth radii. We note that the Apophis 2029 encounter is inside or very close to these suggested thresholds for tidally induced seismicity.

As noted in Section 4, within this White Paper we take no position on any methodology that should be employed or any architecture for executing Earth-based and/or in situ investigations. Entries on methodology in Table I are purely notional in order to demonstrate the range of potential options. Detailed efforts moving forward are needed, both in terms of physical prediction and technological capabilities, to create science definitions for what may be achieved through Apophis 2029 measurements. The Decadal Survey report may consider prescribing the formation of an Apophis 2029 Science Definition Team to discern the achievable objectives. As argued in Section 3, uncertainties in the outcome is a strong driver for making direct measurements of a completely new phenomenon. However, because the uncertainties may include the possibility of no measurable outcome, we recommend science definitions of measurement objectives should include a science value assessment for meaningful constraints gained on the physical nature of PHAs in the event that any attempted investigation yields a null result (signal below the limit of measurement sensitivity). 


\section{Precedents and Lessons in Planetary Science: Applications to Apophis 2029 Planning}

The lower sections in Table I recall the state of predictions in advance of previous "experiments:" the 1994 Jupiter impact of comet Shoemaker-Levy 9 and the 2005 Deep Impact penetration of Tempel 1. The parallels to Apophis 2029 are striking in the considerable range of uncertainties in outcomes up until the moment each "experiment" occurred. The lesson is that only through dedicated planning and measurement can the true outcome be learned, and from that outcome, considerable new insights be gained into the physical nature of planetary bodies. Apophis is coming. Will we be ready? It's up to you to make Apophis 2029 a Decadal priority.

\begin{tabular}{|c|c|c|c|c|}
\hline $\begin{array}{l}\text { Physical Effects } \\
\text { on Apophis }\end{array}$ & $\begin{array}{l}\text { Degree of } \\
\text { Certaintya }\end{array}$ & $\begin{array}{l}\text { Implications of } \\
\text { Measurement }\end{array}$ & \multicolumn{2}{|c|}{$\begin{array}{l}\text { Possible Measurement Methods } \\
\text { Earth-based In situ }\end{array}$} \\
\hline Orbit Change & Certain [F] & $\begin{array}{l}\text { Determine future impact } \\
\text { probabilities. Determine } \\
\text { Yarkovsky effects. }\end{array}$ & $\begin{array}{l}\text { Optical telescopes. } \\
\text { Radar. }\end{array}$ & $\begin{array}{l}\text { Multiple flybys. } \\
\text { Orbiter. } \\
\text { Landed transponder. }\end{array}$ \\
\hline Spin State Change & Certain $[A, C, G, H]$ & $\begin{array}{l}\text { Internal structure, } \\
\text { rigidity. }\end{array}$ & $\begin{array}{l}\text { Optical telescopes. } \\
\text { Radar. }\end{array}$ & $\begin{array}{l}\text { Multiple flybys. } \\
\text { Orbiter. }\end{array}$ \\
\hline $\begin{array}{l}\text { Modification of } \\
\text { Shape or Internal } \\
\text { Structure }\end{array}$ & Unlikely $[A, B, D, H]$ & $\begin{array}{l}\text { Internal structure, } \\
\text { rigidity. }\end{array}$ & $\begin{array}{l}\text { Radar? } \\
\text { ALMA Inter- } \\
\text { ferometry? }\end{array}$ & $\begin{array}{l}\text { Multiple flybys. } \\
\text { Orbiter. } \\
\text { Landed package. }\end{array}$ \\
\hline Seismic Vibrations & $\begin{array}{l}\text { Unlikely }[\mathrm{J}, \mathrm{K}] \\
\text { Uncertain }[\mathrm{D}] \\
\text { Possible }[\mathrm{A}, \mathrm{E}, \mathrm{H}]\end{array}$ & $\begin{array}{l}\text { Interior structure of PHAs. } \\
\text { First seismology of solar } \\
\text { system small body. }\end{array}$ & & $\begin{array}{l}\text { Orbiter? } \\
\text { Landed package. }\end{array}$ \\
\hline $\begin{array}{l}\text { Surface Alterations; } \\
\text { Landslides, regolith } \\
\text { redistribution, boulder } \\
\text { movement. }\end{array}$ & $\begin{array}{l}\text { Unlikely [J] } \\
\text { Possible [A,C,D] }\end{array}$ & $\begin{array}{l}\text { Surface and upper level } \\
\text { structure, cohesiveness. } \\
\text { Possible constraints on } \\
\text { interior structure. }\end{array}$ & $\begin{array}{l}\text { Telescopic spectra? } \\
\text { Radar? } \\
\text { Radiometry? }\end{array}$ & $\begin{array}{l}\text { Multiple flybys. } \\
\text { Orbiter. } \\
\text { Landed package. }\end{array}$ \\
\hline \multirow{2}{*}{$\begin{array}{l}\text { Comet Shoemaker- } \\
\text { Levy } 9 \text { Impact: } \\
\text { Impact Outcomes } \\
\text { Observable? }\end{array}$} & $\begin{array}{l}\text { Unlikely [L]. } \\
\text { "Big Fizzle" }\end{array}$ & $\begin{array}{l}\text { Minor constraints on } \\
\text { comet mass, strength. }\end{array}$ & \multirow{2}{*}{$\begin{array}{l}\text { World-wide, } \\
\text { multi-wavelength, } \\
+ \text { extensive HST. }\end{array}$} & \multirow{2}{*}{$\begin{array}{l}\text { Insufficient time for } \\
\text { dedicated mission. } \\
\text { Galileo successful } \\
\text { inbound observations. }\end{array}$} \\
\hline & $\begin{array}{l}\text { Likely [M]. "Impact } \\
\text { Flash Signatures" }\end{array}$ & $\begin{array}{l}\text { Comet structure, atmos- } \\
\text { phere structure, chemistry. }\end{array}$ & & \\
\hline \multirow{3}{*}{$\begin{array}{l}\text { Deep Impact: } \\
\text { Projectile } \\
\text { Penetration } \\
\text { Outcome }\end{array}$} & $\begin{array}{l}\text { Pass through and } \\
\text { exit other side }[\mathrm{P}]\end{array}$ & $\begin{array}{l}\text { Limiting estimate for low } \\
\text { comet strength \& cohesion. }\end{array}$ & \multirow{3}{*}{$\begin{array}{l}\text { World-wide, } \\
\text { multi-wavelength, } \\
+ \text { extensive HST. }\end{array}$} & \multirow{3}{*}{$\begin{array}{l}\text { Extensive in situ } \\
\text { measurements by } \\
\text { mother spacecraft. }\end{array}$} \\
\hline & $\begin{array}{l}\text { Moderate } \\
\text { penetration }[Q, R]\end{array}$ & $\begin{array}{l}\text { Constrained measures of } \\
\text { comet strength \& cohesion. }\end{array}$ & & \\
\hline & $\begin{array}{l}\text { Minimal } \\
\text { penetration }[Q, R]\end{array}$ & $\begin{array}{l}\text { Limiting estimate for high } \\
\text { comet strength \& cohesion. }\end{array}$ & & \\
\hline
\end{tabular}

TABLE I: Summary of predicted outcomes for the Apophis 2029 encounter, placed in an historical context with predicted outcomes for other "natural" and induced experiments.

aTable References: [A] Scheeres 2005; [B] Holsapple 2006; [C] Souchay 2014; [D] Yu 2014; [E] Garcia 2015 [F] Farnochia 2013; [G] Souchay 2018; [H] DeMartini 2019; [J] Scheeres 2020; [K] Zhang 2020; [L] Weissman 1994; [M] Boslough 1994; [P] Neff 2010; [Q] A’Hearn 2005; [R] Schultz 2005.

${ }^{b}$ Methodologies listed are notional. No specific recommendations on methodologies, or their value assessment, are intended by this White Paper. A dedicated Science Definition Team may be prescribed to produce recommendations.

\section{Acknowledgements}

We thank Mainzer et al. (2020) for setting the broad context of Planetary Defense for the 2023-2032 Decadal Survey. 
Binzel et al. - Apophis 2029

\section{References}

A'Hearn, M. F. et al. (2005). Deep Impact: A Large-Scale Active Experiment on a Cometary Nucleus. Space Science Reviews 117: 1-21.

Barbee, B., et al. (2020). Future Spacecraft Missions for Planetary Defense Preparation. White paper submitted to The Planetary Science and Astrobiology Decadal Survey 2023-2032.

Binzel, R. P. et al. (2010). Earth encounters as the origin of fresh surfaces on near-Earth asteroids. Nature 463: 331-334.

Boslough, M. B. et al. (1994). Mass and penetration depth of Shoemaker-Levy 9 fragments from timeresolved photometry. Geophys. Res. Lett. 21: 1555-1558.

Brozović, M. et al. (2018). Goldstone and Arecibo radar observations of (99942) Apophis in 2012-2013. Icarus 300: 115-128.

Cheng, A. et al. (2020). Apophis 2029 Planetary Defense Mission Options. White paper submitted to The Planetary Science and Astrobiology Decadal Survey 2023-2032.

DeMartini, J. V. et al. (2019). Using a discrete element method to investigate seismic response and spin change of 99942 Apophis during its 2029 tidal encounter with Earth. Icarus 328: 93-103.

Farnocchia, D. et al. (2013). Yarkovsky-driven impact risk analysis for asteroid (99942) Apophis. Icarus 224: 192-200.

Garcia, R. F. et al. (2015). Micro-meteoroid seismic uplift and regolith concentration on kilometric scale asteroids. Icarus 253: 159-168.

Haynes, M. et al. (2020). Asteroids Inside Out: Radar Tomography.

White paper submitted to The Planetary Science and Astrobiology Decadal Survey 2023-2032.

Holsapple, K. et al. (2006). Tidal disruptions: A continuum theory for solid bodies. Icarus 183: 331-348.

Pravec, P. et al. (2014). The tumbling spin state of (99942) Apophis. Icarus 233: 48-60.

Mainzer, A. et al. (2020). The future of Planetary Defense in the era of advanced surveys. White paper submitted to The Planetary Science and Astrobiology Decadal Survey 2023-2032.

Melosh, H. J., chair (2019). Finding Hazardous Asteroids Using Infrared and Visible Wavelength Telescopes. The National Academies Press. https://doi.org/10.17226/25476

MIT Project Apophis (2017). https://eapsweb.mit.edu/mit-project-apophis

Nesvorny', D. et al. (2005). Evidence for asteroid space weathering from the Sloan Digital Sky Survey. Icarus 173: 132-152.

Nesvorny', D. et al. (2010). Do planetary encounters reset surfaces of near-Earth asteroids? Icarus 209: $510-519$.

Neff, T. (2010). From Jars to the Stars: How Ball Came to Build a Comet-Hunting Machine. Earthview Media, Denver, CO, 347 pp.

Popova, O. et al. (2013). Chelyabinsk airburst, damage assessment, meteorite recovery, and characterization. Science 342: 1069-1073.

Robertson, D. K., Mathias, D. L. (2019). Hydrocode simulations of asteroid airbursts and constraints for Tunguska. Icarus 327: 36-47.

Scheeres, D. J. et al. (2005). Abrupt alteration of Asteroid 2004 MN4's [Apophis] spin state during its 2029 Earth flyby. Icarus 178: 281-283.

Scheeres, D. J. et al. (2020). The Abrupt Alteration of Apophis' Spin State and its Implications. Abstract for Apophis T-9 Workshop.

Schultz, P. H. et al. (2005). Expectations for Crater Size and Photometric Evolution from the Deep Impact Collision. Space Sci Rev 117: 207-239.

Souchay, J. et al. (2014). Rotational changes of the asteroid 99942 Apophis during the 2029 close encounter with Earth. Astron. Astrophys. 563: A24.

Souchay, J. et al. (2018). Changes of spin axis and rate of the asteroid (99942) Apophis during the 2029 close encounter with Earth: A constrained model. Astron. Astrophys. 617: A74.

Taylor, P. A. (2020). Ground-Based Radar for Planetary Science and Planetary Defense. White Paper for 2020 Planetary Decadal Survey.

Terentjeva, A., Bakanas, E. (2014). Meteorite producing fragment on the Apophis' orbit. WGN, J. International Meteor Organization 42: 198-200. 2014JIMO...42..198T

Weissman, P. (1994). The big fizzle is coming. Nature 370: 94-95.

$\mathrm{Yu}, \mathrm{Y}$. et al. (2014). Numerical predictions of surface effects during the 2029 close approach of Asteroid 99942 Apophis. Icarus 242: 82-96.

Zhang, Y., Michel, P. (2020). Tidal distortion and disruption of rubble-pile bodies revisited Soft-sphere discrete element analyses. Astron. Astrophys: in press. 